\title{
Angle and phase coding in optical recording
}

\author{
S. F. Pereira ${ }^{a}$, J. F. P. Spronck ${ }^{b}$, V. G. Kutchoukov ${ }^{a}$ and H-A. Bachor ${ }^{c}$ \\ ${ }^{a}$ Optics Research Group, Department of Imaging Science and Technology, \\ Faculty of Applied Sciences, Technical University Delft, \\ Lorentzweg 1, NL-2628 CJ Delft, The Netherlands \\ ${ }^{b}$ Yale University, Department of Astronomy \\ New Haven, CT 06511, USA \\ ${ }^{c}$ Centre for Quantum-Atom Optics, Faculty of Science, \\ The Australian National University, Canberra ACT, 0200 Australia
}

\begin{abstract}
We present simulations and experimental results on encoding information both in the longitudinal and transverse directions of an optical beam reflected from an asymmetric pit. The method does not require interferometric detection but is based on intensity measurements using a simple quadrant detector. In addition, we also discuss the implementation of this scheme in an optical recording setup and make an analysis of the crosstalk between neighboring pits.
\end{abstract}

Keywords: Diffraction and scattering, Fourier optics, Optical storage systems, Optical discs

\section{INTRODUCTION}

In the past few decades, we have seen how the compact disc (CD, DVD) system has revolutionized the area of information storage. This system has proven to be a convenient and non-expensive way to distribute, store and manipulate digital information. Technological advances in recent years have led to an increase on information density on a single layer optical disc of about 34 times if one compares the original CD with the recently developed Blu-Ray system. However, there is still a strong need for exchangeable super high-density, high data rate storage memories that are robust and can be produced at low cost.

The increase in density in optical data storage until now going from the CD to the Blu-Ray systems have been reached by decreasing the focused spot size on the disc, i.e., by reducing the laser wavelength while increasing further the numerical aperture of the focusing system. If the path towards higher densities is to continue this way, the use of wavelengths in the ultraviolet region and extremely high numerical apertures (greater than 1) should be used. Other possibilities for increasing data capacity in optical discs are the use of nonlinear and plasmon effects such as super-RENS, magneto-optic effect, and multilayer storage. ${ }^{1-4}$ Although these techniques have potential to lead to a mature option for optical data storage in future, most of them involve high complexity, limited durability, and high price, which differs in these points considerably from the present compact disc systems. An alternative way to achieve higher spatial densities, i.e., more information per unit area on an optical disc is to explore the technique of multiplexing the information. It consists in encoding more than one bit of information per storage unit. This idea has been explored and proposed in optical recording systems and is based on the fact that the light that is reflected from the pit may contain more than one bit of information that can be stored in, for example, the spatially resolved amplitude, phase, polarization state or angular momentum values. Several schemes and ideas are found in the literature where the use of multiplexing in amplitude (gray level recording), pit depth, multiple wavelengths, induced angular momentum and polarization effects have been explored (for a review on this subejct, see for example ${ }^{5}$ ). Another attractive approach is to consider an asymmetric pit geometry that generates an asymmetric field distribution at the detector. In this way, the orientation of the pit can be used as a multiplexing variable. This is generally referred to as angle multiplexing, and several pit geometries, inclusive the half-circle shape pit discussed in this paper have been introduced in Ref. ${ }^{6}$

Optical Data Storage 2010, edited by Susanna Orlic, Ryuichi Katayama, Proc. of SPIE Vol. 7730, 77300F · C 2010 SPIE · CCC code: 0277-786X/10/\$18 - doi: 10.1117/12.859123 
In this paper, we analyse and demonstrate a multiplexing scheme consisting of asymmetric pits with variable depth and orientation angle. Due to the pit asymmetry and variable pit depth, the signal at the detector varies accordingly with the pit orientation and depth. The detector is a simple quadrant detector. In the experiment, we have used a sequence of pits written with ion beam pattern generator. Based on experimental results and simulations, we believe that this system has potential to be implemented in read-only optical recording systems.

\section{DESCRIPTION OF THE APPROACH}

The multiplexing scheme that we propose relies on writing a basic pit that is not rotationally symmetric and has variable depth so that for each combination of orientation angle and pit depth one bit pattern is assigned. The number of bits which can be assigned in the space of one pit depends on the number of different combinations of angle and pit depth that can be uniquely detected. The pit reading method is however different from that of a usual optical disc; here, we consider a gated system which only records the field that is reflected from the structure at the time interval that the pit is centered with respect to the reading laser beam. As it will be shown below, the retrieval of both orientation and pit depth is done with a quadrant detector. This detection system is not only very simple but also attractive in the sense that the detection is based on differential signal within the laser beam, offering the possibility to extend the detection to the shot-noise level.

Recently, a scheme based on a four different phase front profiles has been suggested by, among others, one of the authors of this paper. ${ }^{7}$ The idea there was to produce a mode at the detector plane that would be compatible with the set of eigenmodes that describe split detectors, and the detection mode was based on an interferometric scheme. Although this idea was interesting in a theoretical point of view, the need of an interferometer to retrieve the pit depth is not attractive for applications in optical data storage. This lead us to consider a new approach, where the interferometric system has been replaced by a simple quadrant detector. In this way, the idea of Ref. ${ }^{7}$ can be considerably improved and simplified as presented in Ref. ${ }^{8}$

The basic pit used in Ref. ${ }^{8}$ is asymmetric and consists of a semi-circle structure such as the one schematically shown in Fig. 1. The angle $\theta$ describes the rotation angle of the pit and $d$ the pit depth. For the analysis of the signal at the detector after the laser has been reflected from the pit, we use the well known optical recording reading system, with the system of the disc readout such as presented in details in Ref. ${ }^{9}$ Using the scalar diffraction approximation, we have that the field distribution at the detector plane $E_{\text {det }}(x, y)$ after interacting with the pit with (complex) reflectivity $r(x, y)$ is given by

$$
E_{\text {det }}(x, y)=\mathcal{F}\left[E_{i n} * \mathcal{F}^{-1}(r)\right](x, y) .
$$

where $\mathcal{F}$ and $\mathcal{F}^{-1}$ denote the Fourier and inverse Fourier transform, respectively. As incident field we consider a diffraction-limited spot (Airy disc) scaled to the wavelength and numerical aperture used in the experiment. The pit has the shape of a semi-circle with orientation angle $\theta$ with respect to the $\mathrm{x}$-axis (track direction) and depth $d$ as schematically shown in Fig. 1a. The diameter of the pit can be varied with respect to the spot size of the laser beam on the disc. The variables used here to define the pit state for a given ratio of the spot size with respect to the pit size are thus the angle $\theta$ of the pit orientation and the phase $\phi=4 \pi d / \lambda$ with $\lambda$ being the wavelength. The extra factor 2 occurs because the pit is on a reflecting surface. A scheme of the arrangement of the quadrant detector is shown in Fig. 1.

By means of simulations we have analysed the intensity distribution of the field at the quadrant detector as a function of the orientation angle and for various values of the pit depth. The intensities that are detected on each segment of the quadrant detector, denoted as $I_{A}, I_{B}, I_{C}$, and $I_{D}$ are further processed by addition and subtraction circuits leading to two resulting signals $I_{1}$ and $I_{2}$ given by:

$$
I_{1}=\left(I_{B}+I_{C}\right)-\left(I_{A}+I_{D}\right) \quad I_{2}=\left(I_{A}+I_{B}\right)-\left(I_{C}+I_{D}\right)
$$

Further, we define the sum signal $I_{s}=I_{A}+I_{B}+I_{C}+I_{D}$ which takes into account the intensity fluctuations of the light beam. 

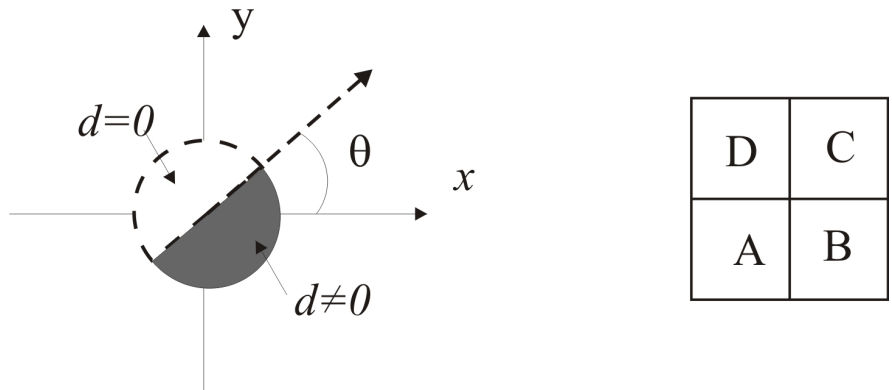

Figure 1. (left) Scheme showing the shape of one single asymmetric pit (shaded area) with orientation angle $\theta$ and depth $d$. The phase change of the shaded area w.r.t. the disc surface after reflection is given by $\phi=4 \pi d / \lambda$. (right) Scheme of the quadrant detector.

In Fig. 2, we show the signals $I_{1}$ and $I_{2}$ as a function of the pit orientation for 3 different pit depths. If one concentrates on the signals $I_{1}$ and $I_{2}$ corresponding to one fixed depth, one can clearly see that the maximum of $I_{1}$ occur when $I_{2}$ is minimum. This means that with the measurement of $I_{1}$ and $I_{2}$, angle orientations varying from 0 to $2 \pi$ can be uniquely distinguished. One can also see that pits with different depths can also be distinguished, since the peak-to-peak intensity is different for each value of the depth. The pit depth corresponding to a total phase shift of $\pi$ has the highest peak-to-peak intensity difference. We have also seen that by changing the ratio denoted by $R$ between the radius of the semi-circle pit and the radius of the focussed spot on the disc, the best discrimination between different states $\theta, \phi$ is achieved when the ratio $R=0.5 .^{8}$

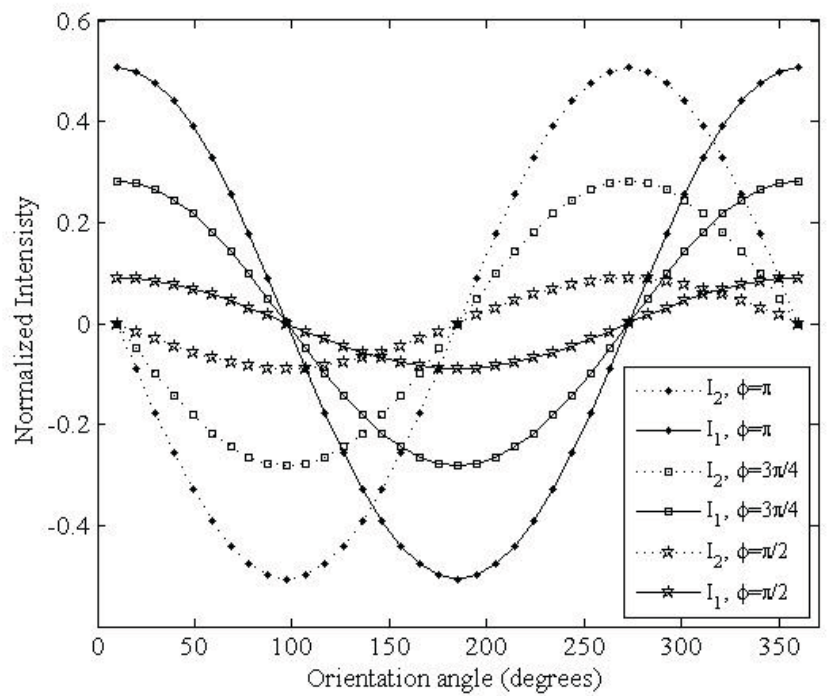

Figure 2. Signals $I_{1}$ and $I_{2}$ as a function of the pit orientation for 3 different pit depths giving rise to phase differences after reflection of $\pi, 3 \pi / 4, \pi / 2$

\section{EXPERIMENTAL SETUP}

We have built an experimental setup in order to test the ideas discussed in the previous section. The general scheme of the setup is shown in Fig. 3 and consists of a quasi static optical recording demonstrator with numerical 
aperture $\mathrm{NA}=0.25$. Since this problem can be scaled to any wavelength, we have chosen, for convenience, a Nd-YAG laser with wavelength of $1064 \mathrm{~nm}$. The numerical aperture can also in principle be scaled to higher values as long as the scalar diffraction theory used in the simulations can still be applied. The laser beam is focussed onto a single mode fiber and its output is collimated with a lens to achieve a beam diameter of about $0.5 \mathrm{~cm}$. In this way, the beam fills the objective aperture and near diffraction limit condition is achieved. The beam that is reflected from the sample passes through the same objective and is directed to a CCD camera. A lens is inserted before the CCD camera to image the disc surface with a magnification of factor of 20 . In order to locate the laser spot relative to the pit, we use a separate LED illumination path (not shown in the figure) configured in such way that simultaneous images of the disc surface and the focussed laser spot are obtained on the CCD camera. The disc has been mounted on top of a $\mathrm{x}-\mathrm{y}-\mathrm{z}$ translator with piezo-electric actuators that are used to align the $x, y, z$ positions between the pits and the laser beam.

The disc was fabricated with an ion beam pattern generator and contains three rows of half-circle and full circle structures written on a silicon wafer. This arrangement was chosen to simulate side tracks, neighboring pits and isolated pits. An image of the disc surface obtained with an electron microscope is shown in Fig. 4. The diameter of the half circles are $5 \mu \mathrm{m}$ and their depth is such that a phase shift $\phi=\pi$ is achieved after reflection for the wavelength of the laser. The full circle structures are used as a one-pit reference. The minimum distance between the pits is $2.5 \mu \mathrm{m}$ and the distance between the tracks (center to center of the pits) is $7.5 \mu \mathrm{m}$. The measured diameter of the laser spot, defined as the distance between the zeros of the Airy disc at the disc surface is $5 \mu \mathrm{m}$.

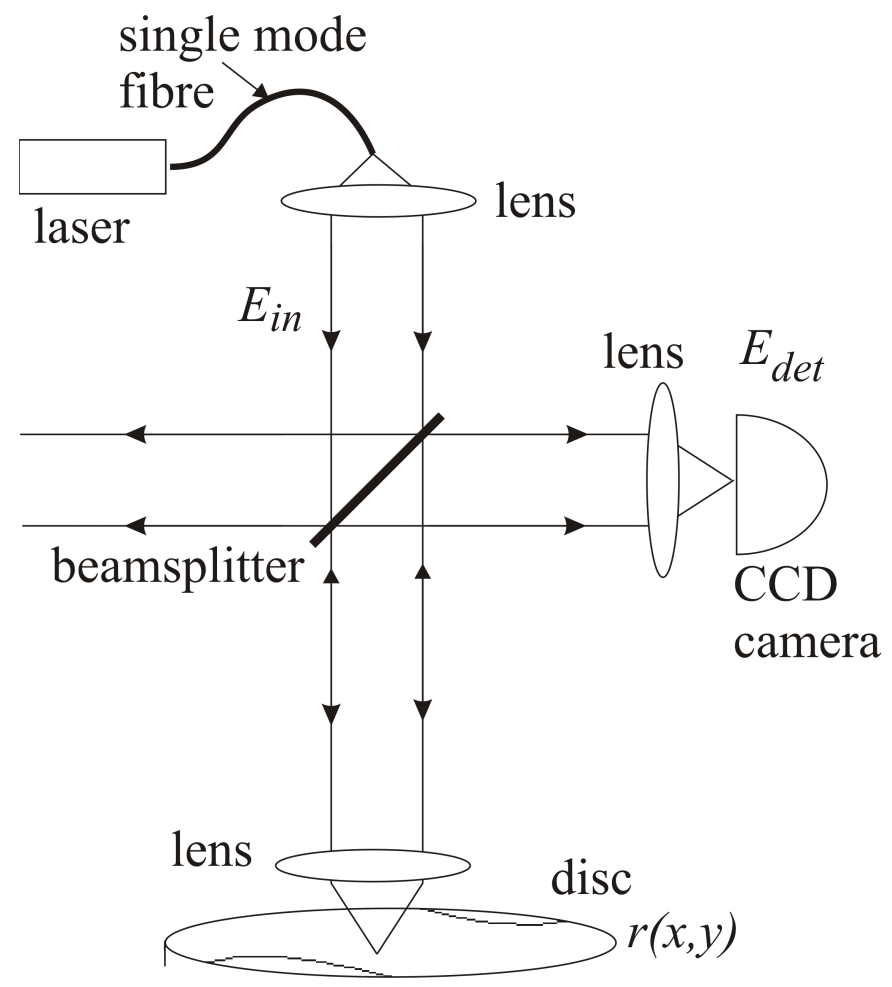

Figure 3. Optical recording system where $\mathrm{E}_{i n}$ and $\mathrm{E}_{\text {det }}$ are the input field and fields at the detector, respectively and $r(x, y)$ is the pit reflectivity 


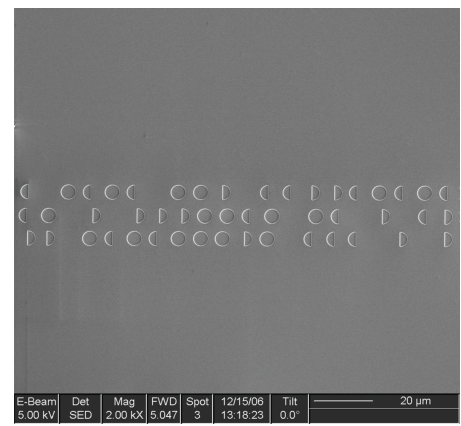

Figure 4. Electron microscope picture showing the sequence of half-circles and full circle structures written on a silicon wafer using an ion beam pattern generator. The diameter of each circle is 5 microns and the depth of the pits is $266 \mathrm{~nm}$.

\section{ANALYSIS OF THE RESULTS}

\subsection{Comparison bertween theory and experiment}

The results obtained in the experiment as described in the previous section were compared with the theoretical expectations. In order to do so, we have directly obtained from the experimental data the relative values of the intensitites of each segment of the CCD camera (simulating thus the intensities $I_{A}, I_{B}, I_{C}, I_{D}$ of the quadrant detector) and calculated the signals $I_{1}$ and $I_{2}$ as well as the sum signal $I_{s}$. In Fig. 5, a comparison between theoretical and experimental values of signals $I_{1}$ and $I_{2}$ as a function of the orientation angle $\theta$ is plotted. The dots and squares in the plot are obtained from the experimental values by rotating the axes of the ABCD quadrants at various angles. The solid lines in Fig. 5 represent results the theoretical curve as in Fig. 2 for the pit depth of $\phi=\pi$ and using the values of the other parameters as in the experiment. As one can see, there is good agreement between theory and experiment. We would like to note that no fitting of the data was done except the normalization of the power.

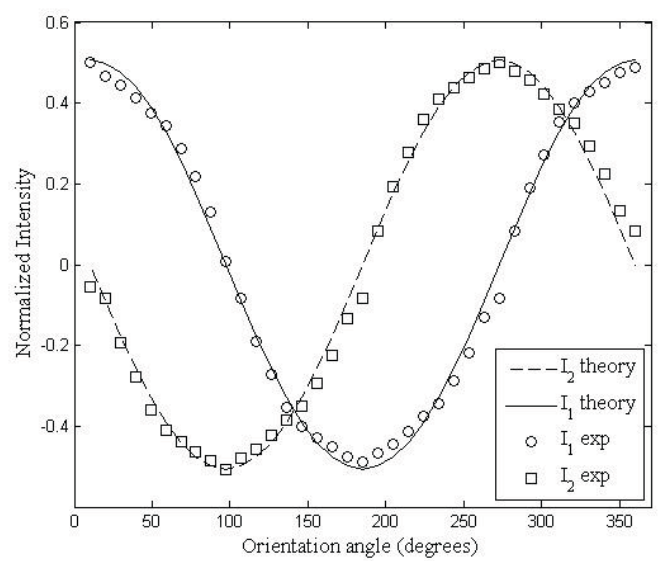

Figure 5. Comparison between experiment and simulation for the signals $I_{1}$ and $I_{2}$ as a function of the orientation angle for the pit phase of $\phi=\pi$ 


\subsection{Considerations about crosstalk}

The experimental data and the comparison between theory and experiment of the previous subsection have been done based on an isolated pit configuration. But for applications in optical recording, the influence of the neighboring pits on the signal due to one pit should be analysed. This is important since it gives a realistic prospect on the information density capability of this system. This factor is usually referred as crosstalk.

We have made simulations to consider the effect of neighboring pits on the signals $I_{1}$ and $I_{2}$. The analysis consisted in considering a central pit surrounded by 4 pits as shown in Fig. 6 and comparing the results with the single pit case. This crosstalk configuration is schematically shown in Fig. 6, and for other orientations of the neighboring pits, the results are about the same.

For the simulations we consider a single pit size of $0.4 \times$ Airy radius and a center-to-center pit distance between pits and between the tracks is $1.18 \times$ Airy radius.

In order to quantify the effect of the neighboring pits on the intensity distribution at the quadrant detector, we calculated the signals denoted as $I_{1 \text { cross }}=I_{1} / I_{s}$ and $I_{2 \text { cross }}=I_{2} / I_{s}$ obtained with the presence of neighboring pits and compared with the signals $I_{1 \text { single }}=I_{1} / I_{s}$ and $I_{2 \text { single }}=I_{2} / I_{s}$ for the case of a single isolated pit. In Fig. 7 we show the signals $I_{2 \text { single }}$ and $I_{2 \text { cross }}$ as a function of the orientation angle $\theta$. By calculating the difference between the two signals $\Delta\left(I_{1}\right)=I_{1 \text { cross }}-I_{1 \text { single }}$ and $\Delta\left(I_{2}\right)=I_{2 \text { cross }}-I_{2 \text { single }}$ for all orientation angles, i.e., for $\theta=0$ to 360 degrees, we have obtained the peak-to-valley deviation of $\Delta\left(I_{1}\right), \Delta\left(I_{2}\right)$.

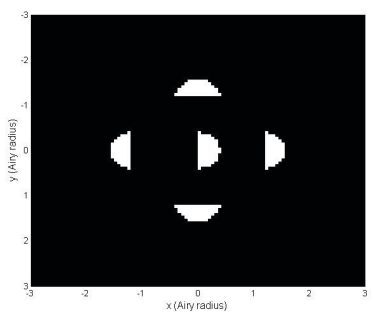

Figure 6. Pit configuration for crosstalk analysis

According to the simulations, for the configuration of Fig. 6 the peak-to-valley intensity variation $\Delta\left(I_{1}\right)=$ $\Delta\left(I_{2}\right)=0.03$. Given the fact that the maximum of $I_{1 \text { single }}=0.3$, we have that the number of intensity levels that can be differentiated is given by $N_{\text {int }}=I_{1 \text { single }} / \Delta\left(I_{1}\right)$ so that one can discriminate $N_{\text {int }}=10$ intensity levels. The total number of states $N$ that can be differentiated has been derived and is given by:

$$
N=2 \times N_{\text {int }}\left(N_{\text {int }}+1\right),
$$

where in the case of $N_{i n t}=10$, the number of states $N=220$. This means that, in terms of bits, $2^{N_{b i t s}}<N$, we have that $N_{\text {bits }}=7$, in other words, one can encode 7 bits within a single pit. Taking the values of $\lambda / \mathrm{NA}$ as in the DVD system, the cell area here will be $0.6 \mu \mathrm{m}^{2}$. Considering the storage of 7 bits per pit area, this will correspond to a density of about 3 times the present single layer DVD system. Note this expected density is relative to the DVD system, but the achievable storage density is scalable with the numerical aperture and wavelength; thus higher densities can be expected when either higher numerical apertures or lower wavelengths are considered.

These calculations give a rough estimate of the prospects of our proposed system. Furhter optimisation of all parameters should be performed, accompanied by experiments in order to obtain a real estimation. This includes optimization of the pit size, the distance between adjacent pits and the distance between tracks. We have also observed that by adding a circular mask on the detector, the error on the signal due to crosstalk can 


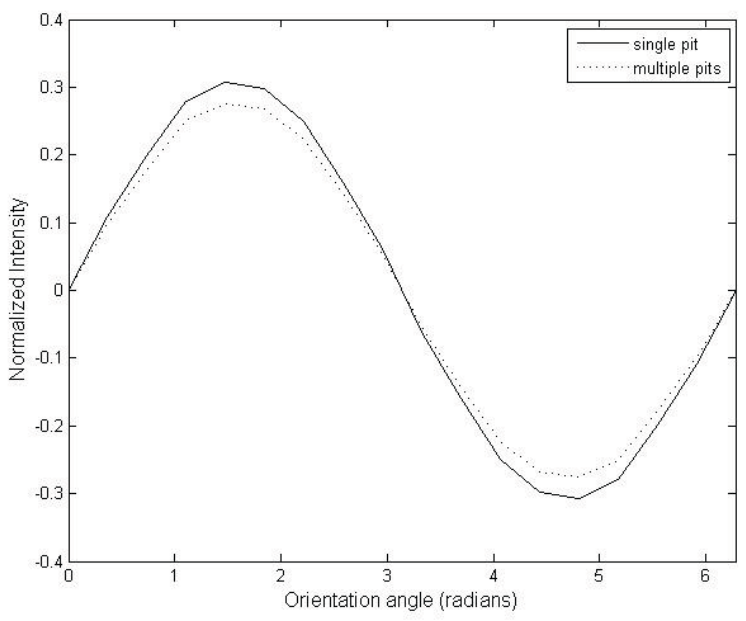

Figure 7. Comparison between the signal $I_{2}$ for the single pit and a pit surrounded with other pits configurations

be minimized. One way to increase further the data density would be to optimize the coding of the information for our system. Since our system allows many bits per pit area, there is a lot of room to extend the studies in this area.

\section{CONCLUSIONS}

In conclusion, we have analyzed a way to multiplex binary bit patterns on one single pit by considering the pit asymmetric and using the orientation and the depth of the pit as encoding variables. We also have shown that the pit depth can be resolved using a single quadrant detector and intensity measurements, without the need of interferometric setup. By performing a crosstalk analysis we have been able to determine ways to minimize the crosstalk and have made an estimate of the information density that can be achieved with this system. Since our method is based on far field detection and uses a quadrant detector, it can be implemented in a quite straightforward way on a present CD/DVD system. This feature offers several advantages of our method as compared to new proposals in optical recording based on near field approach. We also would like to point out that in order to achieve higher densities based on numerical apertures near one (as it is the case of the Blu-Ray system), the present theory based on Fourier optics cannot be applied anymore, so that an extension of the theory to vectorial calculations should be done in order to take polarisation and other diffraction effects into account.

Finally, although we have concentrated this paper in the application of this system in optical recording, other applications can also be considered, as for example metrology systems for alignment of wafers in lithography. In this particular case, one can think on using the reflected signal from the structure of one known asymmetric structure to center a focussed laser spot with respect to the structure and also determine its rotation angle of the wafer.

\section{ACKNOWLEDGMENTS}

S. F. Pereira would like to thank the Center of Excellence ACQAO at the Australian National University for their support and to give the possibility to perform the experiment during her six-months visit. In particular, the help of K. Wagner was much appreciated. V. G. Kutchoukov would like to thank F. Segerink for his assistance in preparing the sample. 


\section{REFERENCES}

1. Tominaga J. and Nakano T 2005 Optical near field recording- science and technology (Springer-Verlag Berlin Heidelberg)

2. Mansuripur M 1995 The physical principles of magneto-optic recording (Cambridge Univ. Press, Cambridge)

3. Mishima K, Yoshitoku D, Itoh H, Kikukawa T, Yamatsu S, Inoue H, Komaki T, Tanaka K, Aoi T 2006150 GB, 6-layer write once disc for Blu-ray disc system Proc. of the SPIE 6282, 628201

4. O'Neill M P and Wong T L 2000 Multi-level data storage using phase-change optical discs Tech. Digest Optical Data Storage 2000, 170

5. van de Nes A S, Braat J J M, and Pereira S F (2006) High density optical data storage Rep. Prog. Phys. 69, 2323 and references herein.

6. Török P, Salt M, Kriezis E E, Munro P R T, Herzig H P, Rockstuhl C, Optical disk and reader therefor, Worldwide Patent No.WO 2006/010882 (2006).

7. Hsu M T L, Delaubert V, Bowen W P, Fabre C, Bachor H A, and Lam P K 2006 A quantum study of multibit phase coding for optical storage IEEE J. of Q. Electron. 42, 1001.

8. Pereira S F, Spronck J F P, Kutchoukov V G and Bachor H. -A 2010 Multiplexing the information on an optical disc by angle and phase coding, J. Opt. 12 No 1, 015402.

9. Bouwhuis G, Braat J J M, Huijser A, Pasman J, van Rosmalen G, and Schouhamer Immink K, 1987 Principles of optical disc systems (Adam Hilger Ldt., Bristol,and Boston), Chap. 2. 